From the International Collaboration of Trialists on behalf of the Medical Research Council Advanced Bladder Cancer Working Party (now the National Cancer Research Institute Bladder Cancer Clinical Studies Group), the European Organisation for Research and Treatment of Cancer GenitoUrinary Tract Cancer Group, the Australian Bladder Cancer Study Group, the National Cancer Institute of Canada Clinical Trials Group, Finnbladder, Norwegian Bladder Cancer Study Group, and Club Urologico Espanol de Tratamiento Oncologico Group. The members and affiliations of the writing committee are listed in the online-only Appendix.

Submitted August 26, 2010; accepted December 21, 2010; published online ahead of print at www.jco.org on April 18, 2011.

The Medical Research Council (MRC) Clinical Trials unit was supported by a grant from the MRC, United Kingdom, and the European Organisation for Research and Treatment of Cancer Data Centre was supported by Grants No. 2 U10 CA11488-19 through 5U10 CA011488-39 from the National Cancer Institute (Bethesda, MD).

The contents of this article are solely the responsibility of the authors and do not necessarily represent the official views of the National Cancer Institute.

Authors' disclosures of potential conflicts of interest and author contributions are found at the end of this article.

Clinical Trials repository link available on JCO.org.

Corresponding author: Gareth Griffiths, CSTAT, Wales Cancer Trials Unit, School of Medicine, Cardiff University, 6th Floor, Neuadd Meirionnydd, Heath Park, Cardiff CF14 4YS, United Kingdom; e-mail: griffithsg@cf.ac.uk.

(c) 2011 by American Society of Clinical Oncology

0732-183X/11/2916-2171/\$20.00

DOI: $10.1200 / J C O .2010 .32 .3139$

\title{
International Phase III Trial Assessing Neoadjuvant Cisplatin, Methotrexate, and Vinblastine Chemotherapy for Muscle-Invasive Bladder Cancer: Long-Term Results of the BA06 30894 Trial
}

International Collaboration of Trialists on behalf of the Medical Research Council Advanced Bladder Cancer Working Party (now the National Cancer Research Institute Bladder Cancer Clinical Studies Group), the European Organisation for Research and Treatment of Cancer Genito-Urinary Tract Cancer Group, the Australian Bladder Cancer Study Group, the National Cancer Institute of Canada Clinical Trials Group, Finnbladder, Norwegian Bladder Cancer Study Group, and Club Urologico Espanol de Tratamiento Oncologico Group

See accompanying editorial on page 2135

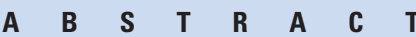

\section{Purpose}

This article presents the long-term results of the international multicenter randomized trial that investigated the use of neoadjuvant cisplatin, methotrexate, and vinblastine (CMV) chemotherapy in patients with muscle-invasive urothelial cancer of the bladder treated by cystectomy and/or radiotherapy. Nine hundred seventy-six patients were recruited between 1989 and 1995, and median follow-up is now 8.0 years.

\section{Patients and Methods}

This was a randomized phase III trial of either no neoadjuvant chemotherapy or three cycles of CMV.

\section{Results}

The previously reported possible survival advantage of CMV is now statistically significant at the $5 \%$ level. Results show a statistically significant $16 \%$ reduction in the risk of death (hazard ratio, $0.84 ; 95 \% \mathrm{Cl}, 0.72$ to $0.99 ; P=.037$, corresponding to an increase in 10 -year survival from $30 \%$ to $36 \%$ ) after $\mathrm{CMV}$.

\section{Conclusion}

We conclude that CMV chemotherapy improves outcome as first-line adjunctive treatment for invasive bladder cancer. Two large randomized trials (by the Medical Research Council/European Organisation for Research and Treatment of Cancer and Southwest Oncology Group) have confirmed a statistically significant and clinically relevant survival benefit, and neoadjuvant chemotherapy followed by definitive local therapy should be viewed as state of the art, as compared with cystectomy or radiotherapy alone, for deeply invasive bladder cancer.

\section{J Clin Oncol 29:2171-2177. (c) 2011 by American Society of Clinical Oncology}

\section{INTRODUCTION}

In 1999, we published the first results of the largest ever randomized trial of neoadjuvant chemotherapy in muscle-invasive bladder cancer. ${ }^{1}$ This was an international multicenter study comparing local radical treatment alone with local radical treatment preceded by three cycles of neoadjuvant cisplatin, methotrexate, and vinblastine (CMV) chemotherapy. The trial was open to patient entry between 1989 and 1995 and recruited 976 patients. The first analysis showed a conventionally nonsignificant $15 \%$ reduction in the risk of death after neoadjuvant chemotherapy (hazard ratio [HR], 0.85; 95\% CI, 0.71 to $1.02 ; P=.075)$, which translated into an absolute difference in 3-year survival of 5.5\% (95\% CI, $-0.5 \%$ to $11 \%$ ), with 3 -year survival rates of $50 \%$ in patients who did not receive CMV and $55.5 \%$ in patients who did receive CMV.

At that time, the median length of follow-up for those patients still alive was 4 years. Also at that time, the survival benefit from neoadjuvant chemotherapy did not meet our prespecified clinical trial goal, and the study was reported as such. The study has now further matured, with a median length of follow-up for patients still alive of more than 8 years. Data for this analysis were locked in 2005. For a number of practical and institutional reasons (eg, centers closing down the trial to further follow-up), only a small amount of more recent data has been 


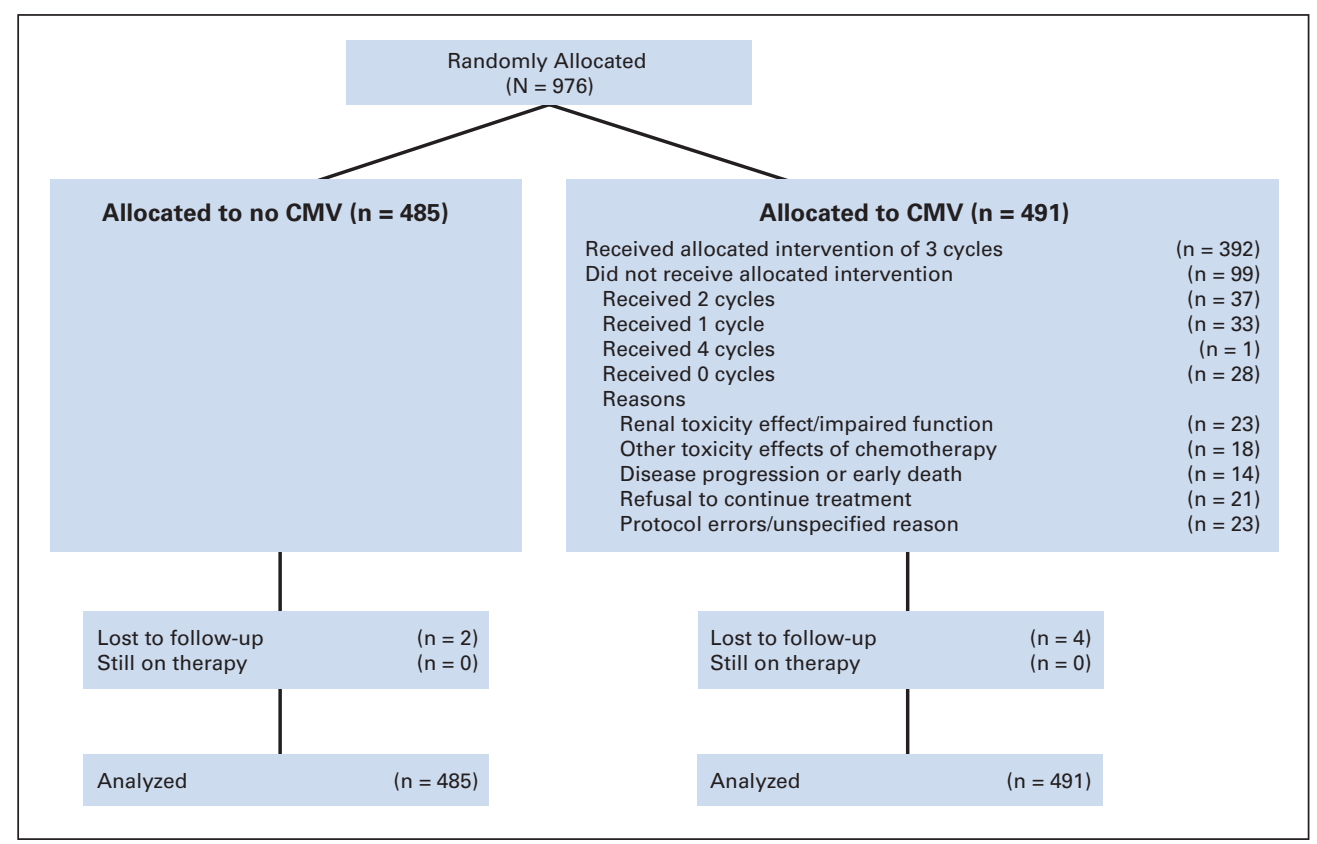

Fig 1. CONSORT diagram. CMV, cisplatin, methotrexate, and vinblastine.

forthcoming, and further analysis with longer follow-up is considered impracticable. The following updated results are presented as the definitive and final outcome of this important study.

\section{PATIENTS AND METHODS}

Full details of the trial design, patient eligibility, and treatment were published previously. ${ }^{1}$ In summary, eligible patients had to have histologically proven muscle-invasive urothelial cell carcinoma of the bladder (T2 grade 3, T3, or T4a and N0/X, M0) and be considered suitable for curative treatment. Glomerular filtration rate had to be more than $50 \mathrm{~mL} / \mathrm{min}$, and informed consent of the patient needed to have been obtained. Figure 1 shows the trial design.

\section{Chemotherapy}

The CMV regimen used in this trial was as follows: day 1: methotrexate $30 \mathrm{mg} / \mathrm{m}^{2}$ intravenous (IV) bolus and vinblastine $4 \mathrm{mg} / \mathrm{m}^{2}$ IV bolus; day 2 before hydration: cisplatin $100 \mathrm{mg} / \mathrm{m}^{2}$ IV infusion; day 2 after hydration: folinic acid $15 \mathrm{mg}$ (oral or IV) every 6 hours for four doses commencing 24 hours after methotrexate on day 1; day 8: methotrexate $30 \mathrm{mg} / \mathrm{m}^{2} \mathrm{IV}$ bolus and vinblastine $4 \mathrm{mg} / \mathrm{m}^{2}$ IV bolus; and day 9: folinic acid $15 \mathrm{mg}$ (oral) every 6 hours for four doses after methotrexate on day 8 . This schedule was repeated every 21 days for a total of three cycles. The protocol contained detailed dose reduction schedules, available on request.

\section{Study Design, Outcome Measures, and Statistical Analysis}

Random assignment was performed by a telephone call, facsimile, or computer connection to the Medical Research Council (MRC) Cancer Trials Office (now the MRC Clinical Trials Unit), European Organisation for Research and Treatment of Cancer Headquarters, National Cancer Institute of Canada Clinical Trials Group Central Office, or Australian National Health and Medical Research Council Clinical Trials Centre. A minimization method for randomly assigning patients was used, and patients were stratified by institution, choice of definitive treatment (cystectomy, radiotherapy, or radiotherapy plus cystectomy) and tumor stage. Each institution selected its preferred local treatment option (radiotherapy/cystectomy) to reduce individual bias in selection of treatments for specific patients.

Kaplan-Meier curves of overall and disease-specific survival, metastases-free survival, locoregional disease-free survival, overall diseasefree survival, and locoregional control were compared using the two-sided log-rank test. All analyses were by intention to treat. To calculate the absolute difference in survival time and the other outcome measures between CMV and no CMV at fixed time points, the HR was applied to the event rate for the no CMV group. ${ }^{2}$

Overall survival was defined as the time from random assignment to death from any cause. Patients still alive were censored at the time of last follow-up. For disease-specific survival, only deaths caused by bladder cancer where considered events. Metastasis-free survival was defined as the

\begin{tabular}{|c|c|c|c|c|c|c|c|c|c|c|c|}
\hline End Point & No CMV & CMV & Difference & $95 \% \mathrm{Cl}$ & No CMV & $\mathrm{CMV}$ & No CMV & CMV & $H R$ & $95 \% \mathrm{Cl}$ & $P$ \\
\hline Metastasis-free survival & 44 & 53 & 9 & 4 to 14 & 38 & 47 & 23 & 33 & 0.77 & 0.66 to 0.90 & .001 \\
\hline Locoregional disease-free survival & 41 & 46 & 5 & 0 to 10 & 34 & 39 & 22 & 27 & 0.87 & 0.75 to 1.01 & .067 \\
\hline Disease-free survival & 38 & 45 & 7 & 2 to 13 & 32 & 39 & 20 & 27 & 0.82 & 0.70 to 0.95 & .008 \\
\hline
\end{tabular}

Abbreviations: CMV, cisplatin, methotrexate, and vinblastine; HR, hazard ratio. 
time from random assignment to first recognition of metastases or death. Patients who were alive and free from metastases were censored at the time of last follow-up. Locoregional disease-free survival was defined as the time from random assignment to reappearance of locoregional disease (invasive tumor within the bladder or pelvis) or death. Patients who were alive and free from locoregional disease were censored at the time of last follow-up. Disease-free survival was defined as the time from random assignment to reappearance of locoregional disease, metastases, or death. Patients who were alive and disease free were censored at the time of last follow-up. Locoregional control was defined as the time from random assignment to reappearance of locoregional disease (invasive tumor within the bladder or pelvis). Patients who were free from locoregional disease were censored at the time of last follow-up.

Exploratory interaction analyses were planned to assess whether CMV was more effective or less effective over no CMV (in terms of overall survival) in subgroups defined by all initial patient characteristics collected at random assignment and the chosen definitive treatment. To test for consistencies in the size of any effect of CMV, a $\chi^{2}$ test for interaction was performed, or when appropriate, a $\chi^{2}$ test for trend was performed. Kaplan-Meier curves of overall survival and locoregional disease-free survival were produced in subgroups of patients who actually received cystec- tomy only and patients who actually received radiotherapy only, and the difference between CMV and no CMV in each subgroup was compared using the two-sided log-rank test.

The trial was originally powered to detect an absolute improvement in 2 -year survival of $10 \%$ (50\% increased to $60 \%$; with a power approaching $90 \%$, a two-sided significance level of $5 \%$ required 374 events). There was no correction of the $P$ value to adjust for the previous analysis.

\section{RESULTS}

Between November 1989 and July 1995, 976 patients were recruited from 106 institutions in 20 countries by seven different national or international clinical groups; 491 patients were randomly assigned to receive $C M V$, and 485 patients were assigned to not receive $C M V$. The initial patient characteristics and choice of local radical treatment have been published previously ${ }^{1}$ and were balanced between arms. In summary, $34 \%(\mathrm{n}=334), 58 \%(\mathrm{n}=567)$, and $8 \%(\mathrm{n}=75)$ of patients had T2, T3, and T4a disease, respectively; $88 \%(\mathrm{n}=854)$ had

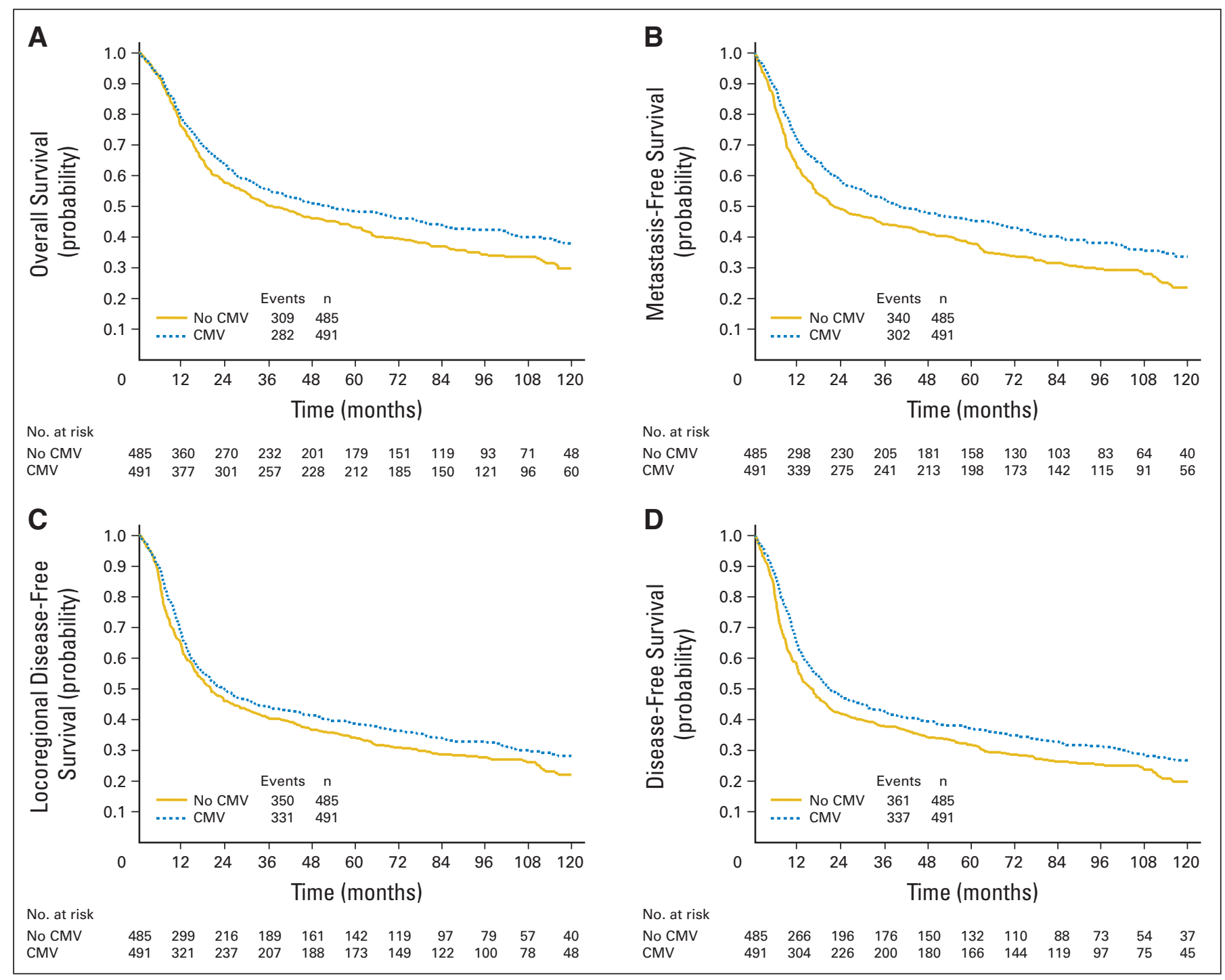

Fig 2. Kaplan-Meier curves for (A) overall survival, (B) metastasis-free survival, (C) locoregional disease-free survival, and (D) disease-free survival. CMV, cisplatin, methotrexate, and vinblastine. 
grade 3 disease; and 65\% $(\mathrm{n}=634)$ had N0. The median age was 64 years; $88 \%$ of patients $(n=863)$ were male; and $43 \%(n=415), 50 \%$ $(\mathrm{n}=485)$, and $8 \%(\mathrm{n}=76)$ of patients chose the local radical treatment of radiotherapy, cystectomy, and a combination of radiotherapy and cystectomy, respectively. In this updated analysis, the median follow-up time for patients still alive is 8.0 years (interquartile range, 5.7 to 10.2 years), and the maximum follow-up time is 13.9 years.

\section{Primary End Points}

The updated results for overall survival, metastases-free survival, locoregional disease-free survival, disease-free survival, and locoregional control are listed in Table 1 and shown in Figure 2. The results show a conventionally statistically significant $16 \%$ reduction in the risk of death (HR, $0.84 ; 95 \% \mathrm{CI}, 0.72$ to $0.99 ; P=.037$ ), $23 \%$ reduction in the risk of metastases or death (HR, $0.77 ; 95 \% \mathrm{CI}, 0.66$ to 0.90 ; $P=.001), 13 \%$ reduction in the risk of local disease or death $(\mathrm{HR}$, $0.87 ; 95 \% \mathrm{CI}, 0.75$ to $1.01 ; P=.067), 18 \%$ reduction in the risk of disease or death (HR, $0.82 ; 95 \% \mathrm{CI}, 0.70$ to $0.95 ; P=.008)$, and $4 \%$ reduction in the risk of locoregional relapse (HR, 0.96 ; 95\% CI, 0.80 to $1.15 ; P=.632)$ after CMV.

\section{Cause of Death}

A total of 591 patients have died (Table 2). The main cause of death was bladder cancer, which was attributed to $72 \%$ of deaths (223 of 309 deaths) in patients who did not receive CMV and 70\% of deaths (198 of 282 deaths) in patient who received CMV. An exploratory analysis of disease-specific survival resulted in an $\mathrm{HR}$ of 0.83 (95\% CI, 0.68 to $1.00 ; P=.050$ ). Table 2 shows cause of death according to the type of definitive treatment actually received.

The addition of CMV to standard treatment resulted in a $16 \%$ reduction in the overall risk of death from all causes. Inevitably, the potential benefit of chemotherapy will have been compromised by any treatment-related deaths. Chemotherapy-related mortality was $1 \%$, operative mortality after cystectomy was $3.7 \%$, and one death was attributed to radiotherapy.

\section{Treatment-Related Mortality}

Trialists were concerned from the outset that the operative risks of cystectomy might be magnified by preoperative chemotherapy or that CMV itself might cause treatment-related deaths, including more cardiovascular toxicity. Table 2 shows that neither treatment was risk free, but chemotherapy-related mortality was low (1\%) and less than half that reported for methotrexate, vinblastine, doxorubicin, and cisplatin (MVAC) in a subsequent international trial of MVAC versus gemcitabine plus cisplatin. ${ }^{3}$

\section{Salvage Chemotherapy}

Twenty-nine patients in the CMV arm and 84 patients in the no CMV arm received some form of salvage chemotherapy. Given the efficacy of chemotherapy observed in this trial and other studies, salvage chemotherapy is unlikely to have influenced overall survival, although it is recognized that the true impact of salvage chemotherapy given in this situation cannot be determined with certainty.

\section{Interaction Analyses}

As reported previously, there is a suggestion (for overall survival) that neoadjuvant CMV may have had a greater effect in the following three subgroups: patients with better renal function, larger tumor size, or poorly differentiated tumors (data not shown). There was no evidence that neoadjuvant CMV had a greater or lesser effect in subgroups of choice of definitive treatment (radiotherapy, cystectomy, or radiotherapy plus cystectomy; test of interaction $\chi^{2}$ value $[d f]=0.112$ $[2] ; P=.946)$.

\section{Radiotherapy Versus Cystectomy}

The choice of definitive treatment used in this trial was based on patient or physician choice and was not randomly assigned, and the trial was designed explicitly not to compare various definitive local treatments. Thus, no conclusions should be drawn from the data presented concerning the relative merit of cystectomy compared with radiotherapy. In Norway, preoperative radiotherapy was carried out, but there were insufficient numbers to analyze separately.

The importance of this caution is endorsed by the finding that although some patient and tumor characteristics were well balanced in the two groups (Table 3), fewer patients receiving radiotherapy, compared with patients who underwent cystectomy, had a WHO performance status of 0 (55\% [223 of 403 patients] $v 82 \%$ [353 of 428 patients], respectively), fewer patients had T2 tumors (31\% [124 of 403 patients] $v 38 \%$ [ 162 of 428 patients], respectively), fewer patients were N0 (62\% [248 of 403 patients] $v 75 \%$ [319 of 428 patients],

\begin{tabular}{|c|c|c|c|c|c|c|c|c|c|c|c|c|}
\hline \multirow[b]{4}{*}{ Cause of Death } & \multicolumn{5}{|c|}{ No CMV (No. of patients) } & \multicolumn{5}{|c|}{ CMV (No. of patients) } & \multirow{3}{*}{\multicolumn{2}{|c|}{$\begin{array}{c}\text { Total } \\
\text { Patients }\end{array}$}} \\
\hline & \multicolumn{4}{|c|}{ Definitive Treatment Received } & \multirow[b]{3}{*}{ Total } & \multicolumn{4}{|c|}{ Definitive Treatment Received } & \multirow[b]{3}{*}{ Total } & & \\
\hline & \multirow[b]{2}{*}{$\mathrm{RT}$} & \multirow{2}{*}{$\begin{array}{c}\text { Preoperative } \\
\text { RT + Cystectomy }\end{array}$} & \multirow[b]{2}{*}{ Cystectomy } & \multirow[b]{2}{*}{ Missing } & & \multirow[b]{2}{*}{$\mathrm{RT}$} & \multirow{2}{*}{$\begin{array}{c}\text { Preoperative } \\
\text { RT }+ \text { Cystectomy }\end{array}$} & \multirow[b]{2}{*}{ Cystectomy } & \multirow[b]{2}{*}{ Missing } & & & \\
\hline & & & & & & & & & & & No. & $\%$ \\
\hline Total patients & 210 & 33 & 212 & 30 & 485 & 193 & 33 & 216 & 49 & 491 & 976 & \\
\hline Patients who died & 144 & 18 & 126 & 21 & 309 & 120 & 14 & 107 & 41 & 282 & 591 & \\
\hline \multicolumn{13}{|l|}{ Cause of death } \\
\hline Disease related (TCC) & 116 & 9 & 80 & 18 & 223 & 93 & 12 & 75 & 18 & 198 & 421 & 71 \\
\hline Treatment related & 0 & 1 & 15 & 0 & 16 & 1 & 0 & 8 & 7 & 16 & 32 & 5 \\
\hline Other malignancy & 8 & 2 & 9 & 0 & 19 & 6 & 0 & 5 & 1 & 12 & 31 & 5 \\
\hline Cardiovascular & 9 & 0 & 11 & 1 & 21 & 9 & 0 & 10 & 9 & 28 & 49 & 8 \\
\hline Other cause & 10 & 5 & 8 & 1 & 24 & 10 & 2 & 6 & 5 & 23 & 47 & 8 \\
\hline Missing & 1 & 1 & 3 & 1 & 6 & 1 & 0 & 3 & 1 & 5 & 11 & 2 \\
\hline
\end{tabular}

Abbreviations: CMV, cisplatin, methotrexate, and vinblastine; RT, radiotherapy; TCC, transitional cell carcinoma. 


\begin{tabular}{|c|c|c|c|c|c|c|}
\hline \multirow[b]{2}{*}{ Characteristic } & \multicolumn{2}{|c|}{$\mathrm{RT}(\mathrm{n}=403)$} & \multicolumn{2}{|c|}{$\begin{array}{c}\text { Preoperative RT }+ \\
\text { Cystectomy }(n=66)\end{array}$} & \multicolumn{2}{|c|}{ Cystectomy ( $n=428$ ) } \\
\hline & No. of Patients & $\%$ & No. of Patients & $\%$ & No. of Patients & $\%$ \\
\hline \multicolumn{7}{|l|}{ Tumor stage } \\
\hline T2 & 124 & 31 & 24 & 36 & 162 & 38 \\
\hline T3 & 256 & 64 & 37 & 56 & 230 & 54 \\
\hline T4a & 23 & 6 & 5 & 8 & 36 & 8 \\
\hline \multicolumn{7}{|c|}{ Histologic grade (local pathologist) } \\
\hline $1 / 2$ & 59 & 15 & 8 & 12 & 45 & 11 \\
\hline 3 & 344 & 85 & 58 & 88 & 382 & 89 \\
\hline Missing & 0 & & 0 & & 1 & \\
\hline \multicolumn{7}{|l|}{ Nodal status } \\
\hline NO & 248 & 62 & 10 & 15 & 319 & 75 \\
\hline NX & 155 & 38 & 56 & 85 & 109 & 25 \\
\hline \multicolumn{7}{|l|}{ Age, years } \\
\hline$<55$ & 55 & 14 & 13 & 20 & 87 & 20 \\
\hline $55-65$ & 151 & 37 & 33 & 50 & 195 & 46 \\
\hline$>65$ & 197 & 49 & 20 & 30 & 146 & 34 \\
\hline \multicolumn{7}{|l|}{ Sex } \\
\hline Male & 348 & 86 & 57 & 86 & 389 & 91 \\
\hline Female & 55 & 14 & 9 & 14 & 39 & 9 \\
\hline \multicolumn{7}{|l|}{ WHO performance status } \\
\hline 0 & 223 & 55 & 48 & 73 & 353 & 82 \\
\hline 1 & 153 & 38 & 18 & 27 & 66 & 15 \\
\hline $2 / 3$ & 27 & 7 & 0 & 0 & 9 & 2 \\
\hline \multicolumn{7}{|l|}{ Tumor size, cm } \\
\hline$\leq 2.5$ & 75 & 19 & 20 & 33 & 66 & 16 \\
\hline $2.6-5.0$ & 247 & 62 & 35 & 53 & 291 & 69 \\
\hline$>5$ & 77 & 19 & 11 & 17 & 66 & 16 \\
\hline Missing & 4 & & 0 & & 5 & \\
\hline \multicolumn{7}{|l|}{ Calculated GFR, $\mathrm{mL} / \mathrm{min}$} \\
\hline$<50$ & 38 & 9 & 2 & 3 & 33 & 8 \\
\hline $50-59$ & 113 & 28 & 17 & 26 & 99 & 23 \\
\hline $60-69$ & 251 & 62 & 47 & 71 & 292 & 69 \\
\hline Missing & 1 & & 0 & & 4 & \\
\hline
\end{tabular}

respectively), and more patients were older than age 65 years (49\% [197 of 403 patients] $v 34 \%$ [146 of 428 patients], respectively). It seems that there was an element of selection when choosing definitive treatment so that survival after these two different forms of treatment should not be compared.

The interaction analysis (previous section) and Figures 3A and 3B show an important finding, namely, that for overall survival, there was no evidence that neoadjuvant CMV was more or less effective when combined with either radiotherapy or cystectomy. A total of 403 patients received radiotherapy alone, and 428 patients received cystectomy alone. The reductions in the risk of death with CMV were 20\% and $26 \%$ for the radiotherapy alone and cystectomy alone groups, respectively (radiotherapy alone: $\mathrm{HR}, 0.80 ; 95 \% \mathrm{CI}, 0.63$ to 1.02 ; $P=.070$; cystectomy alone: $\mathrm{HR}, 0.74 ; 95 \% \mathrm{CI}, 0.57$ to $0.96 ; P=.022$ ).

For locoregional disease-free survival (Figs 3C and 3D), there was some evidence of a greater impact with CMV over no CMV given before cystectomy (a 26\% reduction in risk; HR, 0.74; 95\% CI, 0.58 to $0.95 ; P=.019)$ than the same chemotherapy given before radiotherapy (a 9\% reduction in risk; HR, $0.91 ; 95 \%$ CI, 0.73 to $1.14 ; P=.417$ ). However, this may be explained by the different patient and tumor characteristics discussed earlier.

\section{DISCUSSION}

This analysis of mature data shows that the previously reported possible survival advantage of CMV observed in the first analysis is now statistically significant at the 5\% level and, although the estimates of HRs have remained relatively unchanged, the increase in the number of events has resulted in a narrowing of the $95 \%$ CIs. Three cycles of CMV before cystectomy or radiotherapy results in a $16 \%$ reduction in the risk of death, corresponding to an increase in 3-year survival from $50 \%$ to $56 \%, 10$-year survival from $30 \%$ to $36 \%$, and median survival time of 7 months (from 37 to 44 months).

CMV did not result in more cardiovascular deaths. Some might consider the number of cystectomy-related deaths (3.7\%) to be high, ${ }^{4}$ but this operative mortality rate was lower than that reported in one of the largest reviews of cystectomies performed in the United States ${ }^{5}$ during the years covered by the trial. Thus, there is no evidence to suggest that the addition of neoadjuvant CMV made cystectomy (or radiotherapy) more dangerous in this trial.

At both the outset (1989) and the conclusion (1995) of the trial, more than $70 \%$ of participating clinicians were of the opinion that an 


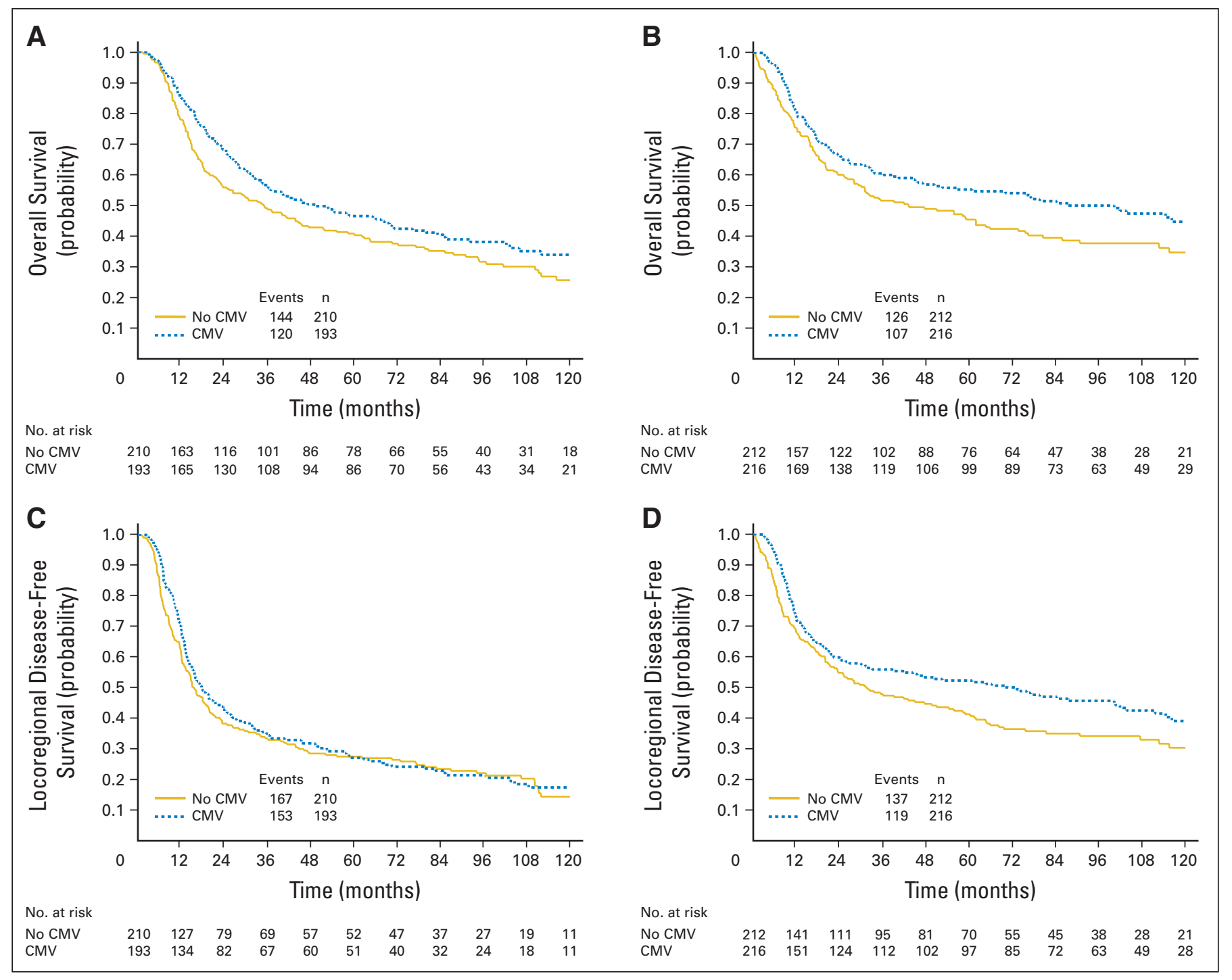

Fig 3. Overall survival in patients who received (A) radiotherapy only and (B) cystectomy only. Locoregional disease-free survival in patients who received (C) radiotherapy only and (D) cystectomy only. CMV, cisplatin, methotrexate, and vinblastine.

improvement in survival of $10 \%$ would be needed to justify the use of neoadjuvant CMV in routine practice. This magnitude of benefit has not been achieved with this trial, which shows, along with the metaanalysis published in 2005 by the Advanced Bladder Cancer Collaboration, ${ }^{6,7}$ a clear benefit in overall survival of only $5 \%$ to $6 \%$ at 3 years.

As is common practice, the end point of locoregional disease-free survival has been defined to include the presence of tumor in the pelvis, distant metastases, and death. This is not helpful from the clinical perspective because the inclusion of distant metastases and death in the definition may overshadow the serious clinical problem of bladder cancer persisting or recurring in the pelvis after completion of definitive treatment. Thus, in Table 2, we have added data for locoregional control (HR, 0.96; 95\% CI, 0.80 to $11.5 ; P=.632)$. These show no significant benefit from the addition of CMV to either radiotherapy or cystectomy, a finding that may be surprising but is important given the high local relapse rate in this situation and its symptomatic consequences for the patient.

Another measure of local control is the number of patients who underwent salvage cystectomy for tumor relapse in the bladder after radiotherapy. It was hoped that the addition of CMV to radiotherapy would improve eradication of primary tumor, but we observed no evidence to support any benefit.

Overall, $49 \%$ of patients ( 240 of 491 patients) who received CMV were reported to have developed locoregional relapse compared with $48 \%$ of patients (231 of 485 patients) who did not receive CMV. In patients who received radiotherapy as definite treatment, $57 \%$ of patients (120 of 210 patients) who received CMV were reported to have developed locoregional relapse compared with $62 \%$ of patients (120 of 193 patients) who did not receive CMV. In patients who received cystectomy as definite treatment, $40 \%$ of patients ( 84 of 212 patients) who received CMV were reported to have developed locoregional relapse compared with 39\% of patients ( 84 of 216 patients) who did not receive CMV.

The Southwest Oncology Group (SWOG) study published in $2003^{8}$ enrolled 317 patients onto a study of neoadjuvant MVAC before cystectomy versus cystectomy alone and showed a $25 \%$ reduction in the risk of death with the addition of MVAC ( $\mathrm{HR}, 0.75 ; 95 \% \mathrm{CI}, 0.57$ to $1.00 ; P=.06)$. In our study, although we have observed a smaller 
reduction in risk of death with CMV (ie, 16\%), this was statistically significant at the 5\% level and included both cystectomy and radiotherapy patients. When analyzing patients in our trial who only had cystectomy ( $\mathrm{n}=428)$, a similar population to that of the SWOG study, we found a similar effect size (HR, 0.74; 95\% CI, 0.57 to 0.96 ; $P=.022$ ), although these results should be interpreted with caution because in our trial the choice of whether to give patients cystectomy or radiotherapy was based on patient or physician choice and was not randomly assigned and there is some evidence to suggest there was an element of patient selection. In the SWOG trial, 33\% of evaluable patients $(n=50)$ had grade 4 (severe) granulocytopenia, with $17 \%$ of patients $(n=26)$ experiencing grade 3 GI toxicity (nausea, vomiting, stomatitis, diarrhea, or constipation). In our study, as reported in the initial analysis, ${ }^{1}$ although serious adverse effects from CMV chemotherapy were not common, five patients assigned to chemotherapy died from toxic effects during treatment (mortality rate, 1\%). Data on nausea and vomiting were not collected, but anecdotal reports suggest that they were common despite the recommended use of antiemetics according to institutional practice. WHO grade 3 or 4 leukopenia, thrombocytopenia, and neutropenic fever occurred in $16 \%, 6.5 \%$, and $10 \%$ of patients, respectively. No grade 3 or 4 renal toxic effects occurred, but $26 \%$ of patients required dose decreases or delay (according to protocol) because of impaired renal function.

How patients and clinicians apply the findings from this trial will vary, and although improvements of this magnitude have resulted in the wide use of adjuvant treatment in a number of other cancers (including breast, colon, ovary, and stomach), the increase in survival will need to be balanced against the toxicity and other disadvantages of chemotherapy (eg, the cost to the patient in terms of treatment time and impact on quality of life). This is a decision each doctor has to make with each patient.

Although the combination of gemcitabine with cisplatin has shown comparable efficacy and lesser toxicity when compared with MVAC, ${ }^{3}$ no new agent or combination has yet shown any evidence of being more active or effective than CMV or MVAC. Although reduced morbidity is welcome, it is significantly increased efficacy that is required. Also of importance, no randomized trial has shown that gemcitabine plus cisplatin confers an equivalent survival benefit in the neoadjuvant context.
Since completion of the trial in 1995, other chemotherapy regimens have been introduced for advanced urothelial cancer, ${ }^{9-11}$ and some authors have suggested that they should be tested in new trials of adjuvant or neoadjuvant therapy. It would seem that although overall response rates of $50 \%$ to $60 \%$ and complete response rates of $20 \%$ may be encouraging indicators of activity in phase II studies, they may not predict a major increment in cure rate in phase III studies in the (neo)adjuvant setting, and it is likely that only modest improvements will be achieved. Therefore, to ultimately get considerable improvements for both individuals and populations, a series of modest improvements to be added together may be required, as opposed to one breakthrough treatment. We believe that future trials should consider new strategies for treatment development such as the development of new surrogate end points/biomarkers and targeted agents that may provide better evidence of treatment activity and individualized treatment strategies that may result in larger improvements in specific subpopulations.

We conclude that CMV chemotherapy improves outcome as firstline adjunctive treatment for invasive bladder cancer. Neoadjuvant CMV or MVAC chemotherapy followed by definitive local therapy should constitute the state of the art for fit patients with deeply invasive bladder cancer, as compared with cystectomy or radiotherapy alone.

\section{AUTHORS' DISCLOSURES OF POTENTIAL CONFLICTS} OF INTEREST

The author(s) indicated no potential conflicts of interest.

\section{AUTHOR CONTRIBUTIONS}

Conception and design: Reginald Hall, Richard Sylvester, Derek

Raghavan, Mahesh K.B. Parmar

Provision of study materials or patients: Reginald Hall

Collection and assembly of data: Richard Sylvester, Derek Raghavan, Mahesh K.B. Parmar

Data analysis and interpretation: Gareth Griffiths, Richard Sylvester, Derek Raghavan, Mahesh K.B. Parmar

Manuscript writing: All authors

Final approval of manuscript: All authors

\section{REFERENCES}

1. International Collaboration of Trialists: Neoadjuvant cisplatin, methotrexate and vinblastine chemotherapy for muscle-invasive bladder cancer: A randomised controlled trial. Lancet 354:533-540, 1999

2. Parmar MKB, Machin D: Survival Analysis: A Practical Approach. Chichester, United Kingdom, John Wiley \& Sons, 1995

3. von der Maase $H$, Sengelov $L$, Roberts JT, et al: Long-term survival results of a randomized trial comparing gemcitabine plus cisplatin, with methotrexate, vinblastine, doxorubicin, plus cisplatin in patients with bladder cancer. J Clin Oncol 23:4602-4608, 2005
4. Rosario DJ, Becker M, Anderson JB: The changing pattern of mortality and morbidity from radical cystectomy. BJU Int 85:427-430, 2000

5. Hollenbeck BK, Wei Y, Birkmeyer JD: Volume, process of care, and operative mortality for cystectomy for bladder cancer. Urology 69:871-875, 2007

6. Advanced Bladder Cancer Meta-Analysis Collaboration: Neoadjuvant chemotherapy in invasive bladder cancer: Update of a systematic review and meta-analysis of individual patient data. Eur Urol 48:202-206, 2005

7. Advanced Bladder Cancer Meta-Analysis Collaboration: Adjuvant chemotherapy in invasive bladder cancer: A systematic review and meta-analysis of individual patient data. Eur Urol 48:189-201, 2005
8. Grossman HB, Natale RB, Tangen CM, et al: Neoadjuvant chemotherapy plus cystectomy compared with cystectomy alone for locally advanced bladder cancer. N Engl J Med 349:859-866, 2003

9. Stenzl A, Cowan NC, De Santis M, et al: The updated EAU guidelines on muscle-invasive and metastatic bladder cancer. Eur Urol 55:815-825, 2009

10. Pliarchopoulou K, Laschos K, Pectasides D: Current chemotherapeutic options for the treatment of advanced bladder cancer: A review. Urol Oncol [epub ahead of print on September 13, 2010]

11. Volpe A, Racioppi M, D'Agostino D, et al: Advanced bladder cancer: New agents and new approaches-A review. Urol Oncol [epub ahead of print on September 21, 2010] 